\title{
Quadrupole Phonons in the Cadmium Isotopes
}

\author{
A. Leviatan $*$ and N. Gavrielov \\ Racah Institute of Physics, The Hebrew University, Jerusalem 91904, Israel \\ J.E. García-Ramo: \\ Departamento de Ciencias Integradas y Centro de Estudios Avanzados en Fúsica, \\ Matemática y Computación, Universidad de Huelva, 21071 Huelva, Spain and \\ Instituto Carlos I de Física Teórica y Computacional, \\ Universidad de Granada, Fuentenueva s/n, 18071 Granada, Spain \\ P. Van Isacker \$ \\ Grand Accélérateur National d'Ions Lourds, CEA/DRF-CNRS/IN2P3, Bvd Henri Becquerel, F-14076 Caen, France
}

(Dated: October 24, 2018)

\begin{abstract}
A key question concerning the spherical-vibrator attributes of states in Cadmium isotopes is addressed by means of a boson Hamiltonian encompassing U(5) partial dynamical symmetry. The $\mathrm{U}(5)$ symmetry is preserved in a segment of the spectrum and is broken in particular non-yrast states, and the resulting mixing with the intruder states is small. The vibrational character is thus maintained in the majority of low-lying normal states, as observed in ${ }^{110} \mathrm{Cd}$.
\end{abstract}

PACS numbers: 21.60.Fw, 21.10.Re, 21.60.Ev, 27.60.+j

The concept of a phonon is indispensable to understand collective behavior in quantum-mechanical manybody systems. In particular in condensed matter, the description of lattice excitations requires the introduction of elementary modes of vibration that are identified with phonons. Phonons also play a central role in nuclear physics, notably in the interpretation of the collective motion of nucleons in an atomic nucleus. A standard model of the nucleus is in terms of a quantum liquid drop that exhibits vibrations around an equilibrium shape, which, if deformed, can also rotate. In their seminal studies Bohr and Mottelson [1 3 ] argued that the collective low-energy properties of nuclei are dominated by quadrupole vibrations, whose nature depends on the equilibrium shape. Small oscillations about spherical equilibrium can be described in terms of a single type of quadrupole phonon while the oscillations about a quadrupole-deformed equilibrium require the introduction of two different phonons that generate so-called $\beta$ and $\gamma$ vibrations.

This Rapid Communication deals with vibrations of spherical nuclei. The first observation to be made is that, despite more than half a century of research, the phonon interpretation of low-energy nuclear structure remains controversial, as exemplified by the Cadmium isotopes. The latter since long have been considered as archetypal examples of nuclei that exhibit small-amplitude vibrations around a spherical shape, to the extent that they have become textbook material to illustrate nuclear phonon behavior [3 7]. Evidence for near-harmonic vi-

\footnotetext{
* ami@phys.huji.ac.il

$\dagger$ noam.gavrielov@mail.huji.ac.il

$\ddagger$ enrique.ramos@dfaie.uhu.es

$\S \overline{\text { isacker@ganil.fr }}$
}

brational properties of $\mathrm{Cd}$ isotopes was reported for up to three [8] and even up to six [9] quadrupole phonons. Nevertheless, it was also realized early on [10] that not all low-energy levels of these isotopes can be considered as vibrational and that additional levels exist at low excitation energies. The latter, named coexisting or intruder states [11, were claimed to arise because of proton excitations across the $Z=50$ shell closure, character that was later confirmed in two-proton transfer reactions [12]. Over the years intruder bands were identified in many even-mass Cd isotopes [13] and, in parallel, models were extended to include such states. Extensive E2 decay patterns were established in several $\mathrm{Cd}$ isotopes and reproduced theoretically, albeit laboriously, by allowing mixing between vibrational and intruder states, see e.g. Refs. [14, 15]. However, as more data on the Cd isotopes were collected, the interpretation in terms of vibrationintruder mixing became increasingly untenable: decay properties of ${ }^{112} \mathrm{Cd}$ could not be explained [16, those of ${ }^{114} \mathrm{Cd}$ were found to be "enigmatic" [17, of ${ }^{116} \mathrm{Cd}$ to be "puzzling" 18. The crisis culminated in papers claiming the "breakdown of vibrational motion" not only in the $\mathrm{Cd}[19$ but also in the neighboring $\mathrm{Pd}$ and $\mathrm{Sn}$ isotopes [20. This paradoxical behavior, characterized in 11] as an unsolved problem, continues to attract considerable attention [21 27].

In this Rapid Communication, we suggest that the vibrational interpretation of the $\mathrm{Cd}$ isotopes can be resurrected not, as attempted previously, by mixing vibrational and intruder states but by mixing particular phonon states. From a formal point of view, the latter mechanism represents a departure from $\mathrm{U}(5)$, which is the dynamical symmetry (DS) of spherical nuclei in the collective model [3] and the interacting boson model (IBM) 4, and generalizes it to a U(5) partial dynamical symmetry (PDS) 28]. 


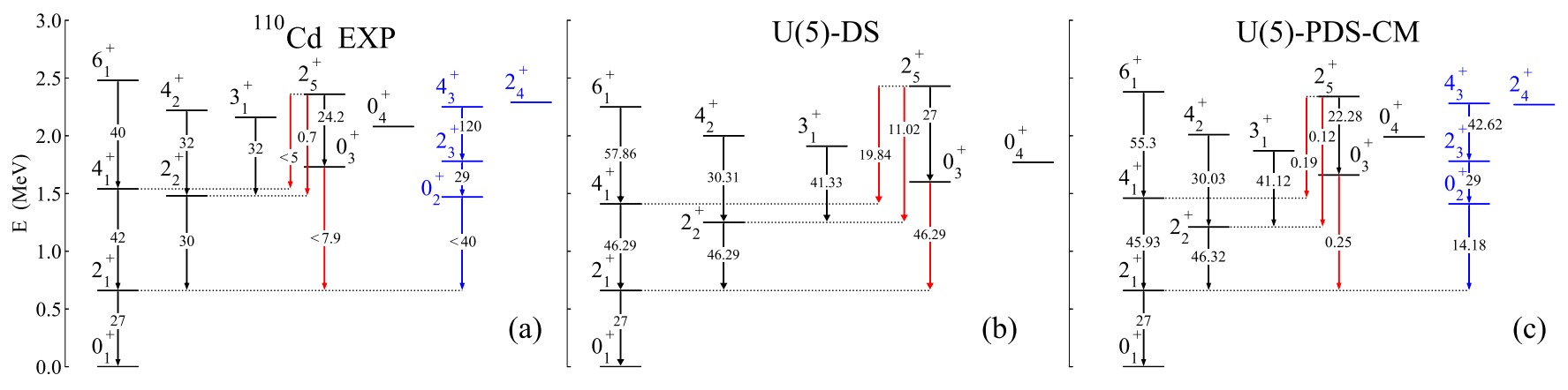

FIG. 1. (a) Experimental spectrum and representative $E 2$ rates [21, 36] (in W.u.) of normal and intruder levels $\left(0_{2}^{+}, 2_{3}^{+}, 4_{3}^{+}, 2_{4}^{+}\right.$) in ${ }^{110} \mathrm{Cd}$. (b) Calculated U(5)-DS spectrum obtained from $\hat{H}_{\mathrm{DS}}[2]$ with parameters $t_{1}=715.75, t_{2}=-t_{3}=42.10, t_{4}=11.38 \mathrm{keV}$ and $N=7$. (c) Calculated U(5)-PDS-CM spectrum, obtained from $\hat{H} 11$ with parameters $t_{1}=767.83, t_{2}=-t_{3}=73.62, t_{4}=$ 18.47, $r_{0}=2.15, e_{0}=-6.92, \kappa=-72.73, \Delta=9978.86, \alpha=-42.78 \mathrm{keV}$ and $N=7(9)$ in the normal (intruder) sector. For a complete listing of $B(E 2)$ values and choice of $E 2$ parameters, see Tables I-II.

A Hamiltonian with DS is written as a linear combination of Casimir operators of nested algebras, leading to complete solvability of its spectrum with exact quantum numbers for all eigenstates [4, 29]. This property, although very appealing, is rarely, if ever, satisfied in an existing quantum-mechanical system. However, more realistic Hamiltonians can be constructed, which satisfy the stringent DS conditions only partially. This leads to three different types of PDS: (i) some eigenstates retain all quantum numbers 30, 31, (ii) all eigenstates retain some quantum numbers [32, 33, and (iii) some eigenstates retain some quantum numbers [34.

In the following we apply a PDS of type (i) to explain the spectroscopic properties of ${ }^{110} \mathrm{Cd}$. The starting point is the $\mathrm{U}(5)$ limit of the IBM, corresponding to the following chain of nested algebras [4, 35],

$$
\mathrm{U}(6) \supset \mathrm{U}(5) \supset \mathrm{SO}(5) \supset \mathrm{SO}(3) \text {. }
$$

The basis states $\left|[N], n_{d}, \tau, n_{\Delta}, L\right\rangle$ have quantum numbers which are the labels of irreducible representations of the algebras in the chain. Here $N$ is the total number of monopole $(s)$ and quadrupole $(d)$ bosons, $n_{d}$ and $\tau$ are the $d$-boson number and seniority, respectively, and $L$ is the angular momentum. The multiplicity label $n_{\Delta}$ counts the maximum number of $d$-boson triplets coupled to $L=0$. The $\mathrm{U}(5)$-DS Hamiltonian has the form

$$
\hat{H}_{\mathrm{DS}}=t_{1} \hat{n}_{d}+t_{2} \hat{n}_{d}^{2}+t_{3} \hat{C}_{\mathrm{SO}(5)}+t_{4} \hat{C}_{\mathrm{SO}(3)}
$$

where $\hat{C}_{\mathrm{G}}$ denotes a Casimir operator of $\mathrm{G}$, and $\hat{n}_{d}=\sum_{m} d_{m}^{\dagger} d_{m}=\hat{C}_{\mathrm{U}(5)} . \quad \hat{H}_{\mathrm{DS}}$ is completely solvable for any choice of parameters $t_{i}$, with eigenstates $\left|[N], n_{d}, \tau, n_{\Delta}, L\right\rangle$ and eigen-energies

$$
E_{\mathrm{DS}}=t_{1} n_{d}+t_{2} n_{d}^{2}+t_{3} \tau(\tau+3)+t_{4} L(L+1) .
$$

A typical U(5)-DS spectrum exhibits $n_{d}$-multiplets of a spherical vibrator, with a two-phonon $\left(n_{d}=2\right)$ triplet of states $(L=4,2,0)$ at an energy $E\left(n_{d}=2\right) \approx 2 E\left(n_{d}=1\right)$ above the ground state $\left(n_{d}=L=0\right)$ and first-excited state $\left(n_{d}=1, L=2\right)$, and a three-phonon $\left(n_{d}=3\right)$ quintuplet of states $(L=6,4,3,0,2)$ at $E\left(n_{d}=3\right) \approx 3 E\left(n_{d}=1\right)$. A quadrupole operator proportional to

$$
\hat{Q}=d^{\dagger} s+s^{\dagger} \tilde{d}
$$

enforces strong $\left(n_{d}+1 \rightarrow n_{d}\right) E 2$ transitions with particular ratios, e.g., $\frac{B\left(E 2 ; n_{d}=2, L=0,2,4 \rightarrow n_{d}=1, L=2\right)}{B\left(E 2 ; n_{d}=1, L=2 \rightarrow n_{d}=0, L=0\right)}=2 \frac{(N-1)}{N}$.

The empirical spectrum of ${ }^{110} \mathrm{Cd}$, shown in Fig. 1(a), consists of both normal and intruder levels, the latter based on $2 \mathrm{p}-4 \mathrm{~h}$ proton excitations across the $Z=50$ closed shell. Experimentally known $E 2$ rates are listed in Tables I and II. A comparison of the calculated spectrum [Fig. (1b)] and $B(E 2)$ values [Table I], obtained from $\hat{H}_{\mathrm{DS}}$ 2 , demonstrates that most normal states have good spherical-vibrator properties, and conform well with the properties of U(5)-DS. However, the measured rates for $E 2$ decays from the non-yrast states, $0_{3}^{+}\left(n_{d}=2\right)$ and $\left[0_{4}^{+}, 2_{5}^{+}\left(n_{d}=3\right)\right]$, reveal marked deviations from this behavior. In particular, $B\left(E 2 ; 0_{3}^{+} \rightarrow\right.$ $\left.2_{1}^{+}\right)<7.9, B\left(E 2 ; 2_{5}^{+} \rightarrow 4_{1}^{+}\right)<5, B\left(E 2 ; 2_{5}^{+} \rightarrow 2_{2}^{+}\right)=0.7_{-0.6}^{+0.5}$ W.u., are extremely small compared to the U(5)-DS values: 46.29, 19.84, 11.02 W.u., respectively. Absolute $B(E 2)$ values for transitions from the $0_{4}^{+}$state are not known, but its branching ratio to $2_{2}^{+}$is small.

Attempts to explain the above deviations in terms of mixing between the normal spherical [U(5)-like] states and intruder deformed [SO(6)-like] states have been shown to be unsatisfactory [19, 21]. The reasons are twofold. (i) An adequate description of the two-phonon $0_{3}^{+}$ state requires strong (maximal $\sim 50 \%$ ) normal-intruder mixing which, in turn, results in serious disagreements with the observed decay pattern of three-phonon yrast states. (ii) The discrepancy in the decays of the nonyrast two- and three-phonon states persists throughout the range $A=110-126$, including the heavier ${ }^{A} \mathrm{Cd}$ isotopes [22, 23], even though the energy of intruder states rises away from neutron mid-shell, and the mixing is reduced. These observations have led to the conclusion that the normal-intruder strong-mixing scenario needs to be rejected, and have raised serious questions on the valid- 
TABLE I. Absolute (relative in square brackets) $B(E 2)$ values in W.u. for $E 2$ transitions from normal levels in ${ }^{110} \mathrm{Cd}$. The experimental (EXP) values are taken from [21, 36. The U(5)-DS values are obtained for an $E 2$ operator $e_{B} \hat{Q}$, Eq. (4), with $e_{B}=1.964$ (W.u. $)^{1 / 2}$. The U(5)-PDS-CM values are obtained using $\hat{T}(E 2)$, Eq. 13 , with $e_{B}^{(N)}=1.956$ and $e_{B}^{(N+2)}=1.195$ (W.u. $)^{1 / 2}$. In both calculations the boson effective charges were fixed by the empirical $2_{1}^{+} \rightarrow 0_{1}^{+}$rate. Intruder states $0_{2 ; i}^{+} 2_{3 ; i}^{+}, 4_{3 ; i}^{+}, 2_{4 ; i}^{+}$, are marked by a subscript $i$.

\begin{tabular}{|c|c|c|c|c|}
\hline$L_{i}$ & $L_{f}$ & EXP & $\mathrm{U}(5)-\mathrm{DS}$ & $\mathrm{U}(5)-\mathrm{PDS}-\mathrm{CM}$ \\
\hline $2_{1}^{+}$ & $0_{1}^{+}$ & $27.0(8)$ & 27.00 & 27.00 \\
\hline $4_{1}^{+}$ & $2_{1}^{+}$ & $42(9)$ & 46.29 & 45.93 \\
\hline \multirow[t]{2}{*}{$2_{2}^{+}$} & $2_{1}^{+}$ & $30(5) ; 19(4)^{a}$ & 46.29 & 46.32 \\
\hline & $0_{1}^{+}$ & $1.35(20) ; 0.68(14)^{a}$ & 0.00 & 0.00 \\
\hline \multirow[t]{2}{*}{$0_{3}^{+}$} & $2_{2}^{+}$ & $<1680^{a}$ & 0.00 & 55.95 \\
\hline & $2_{1}^{+}$ & $<7.9^{a}$ & 46.29 & 0.25 \\
\hline \multirow[t]{3}{*}{$6_{1}^{+}$} & $4_{1}^{+}$ & $40(30) ; 62(18)^{a}$ & 57.86 & 55.30 \\
\hline & $4_{2}^{+}$ & $<5^{a}$ & 0.00 & 0.00 \\
\hline & $4_{3 ; i}^{+}$ & $14(10) ; 36(11)^{a}$ & & 2.39 \\
\hline \multirow[t]{4}{*}{$4_{2}^{+}$} & $4_{1}^{+}$ & $12_{-6}^{+4} ;{ }^{a} 10.7_{-4.8}^{+4.9}$ & 27.55 & 27.45 \\
\hline & $2_{2}^{+}$ & $32_{-14}^{+10} ; 22(10)^{a}$ & 30.31 & 30.03 \\
\hline & $2_{1}^{+}$ & $0.20_{-0.09}^{+0.06} ; 0.14(6)^{a}$ & 0.00 & 0.00 \\
\hline & $2_{3 ; i}^{+}$ & $<0.5^{a}$ & & 0.005 \\
\hline \multirow[t]{4}{*}{$3_{1}^{+}$} & $4_{1}^{+}$ & $5.9_{-4.6}^{+1.8} ;{ }^{a} 2.4_{-0.8}^{+0.9}$ & 16.53 & 16.48 \\
\hline & $2_{2}^{+}$ & $32_{-24}^{+8} ; 22.7(69)^{a}$ & 41.33 & 41.12 \\
\hline & $2_{1}^{+}$ & $1.1_{-0.8}^{+0.3} ; 0.85(25)^{a}$ & 0.00 & 0.00 \\
\hline & $2_{3 ; i}^{+}$ & $<5^{a}$ & & 0.012 \\
\hline \multirow[t]{3}{*}{$0_{4}^{+}$} & $2_{2}^{+}$ & {$\left[<0.65^{a}\right]$} & 57.86 & 1.24 \\
\hline & $2_{1}^{+}$ & {$\left[0.010^{a}\right]$} & 0.00 & 31.76 \\
\hline & $2_{3 ; i}^{+}$ & {$\left[100^{a}\right]$} & & 16.32 \\
\hline \multirow[t]{6}{*}{$2_{5}^{+}$} & $0_{3}^{+}$ & $24.2(22)^{a}$ & 27.00 & 22.28 \\
\hline & $4_{1}^{+}$ & $<5^{a}$ & 19.84 & 0.19 \\
\hline & $2_{2}^{+}$ & ${ }^{a} 0.7_{-0.6}^{+0.5}$ & 11.02 & 0.12 \\
\hline & $2_{1}^{+}$ & $2.8_{-1.0}^{+0.6}$ & 0.00 & 0.00 \\
\hline & $2_{3 ; i}^{+}$ & $<5^{a}$ & & 0.002 \\
\hline & $0_{2 ; i}^{+}$ & $<1.9^{a}$ & & 0.20 \\
\hline
\end{tabular}

a From Ref. 21]

ity of the multi-phonon interpretation 19, 21. In what follows, we consider a possible explanation for the " $\mathrm{Cd}$ problem", based on U(5)-PDS. The latter corresponds to a situation in which the U(5)-DS is obeyed by only a subset of states and is broken in other states. Similar PDS-based approaches have been implemented in nuclear spectroscopy, in conjunction with the SU(3)-DS 31, 3739] and SO(6)-DS [33, 34, 40, chains of the IBM. Here we focus on U(5)-PDS associated with the chain (1).

The lowest spherical-vibrator levels comprise three classes of states,

$$
\begin{array}{lll}
\text { Class A : } & n_{d}=\tau=0,1,2,3 & \left(n_{\Delta}=0\right), \\
\text { Class B : } & n_{d}=\tau+2=2,3 & \left(n_{\Delta}=0\right), \\
\text { Class C : } & n_{d}=\tau=3 & \left(n_{\Delta}=1\right) .
\end{array}
$$

In the U(5)-DS calculation of Fig 1(b), applicable to
TABLE II. $B(E 2)$ values (in W.u.) for $E 2$ transitions fron $\boldsymbol{q}^{2}$ intruder levels in ${ }^{110} \mathrm{Cd}$. Notation and relevant information on the observables shown, are as in Table I.

\begin{tabular}{lllc}
\hline$L_{i}$ & $L_{f}$ & EXP & U(5)-PDS-CM \\
\hline $0_{2 ; i}^{+}$ & $2_{1}^{+}$ & $<44^{a}$ & 14.18 \\
$2_{3 ; i}^{+}$ & $0_{2 ; i}^{+}$ & $29(5)^{a}$ & 29.00 \\
& $0_{1}^{+}$ & $0.31_{-0.12}^{+0.08} ; 0.28(4)^{a}$ & 0.08 \\
& $2_{1}^{+}$ & $0.7_{-0.4}^{+0.3} ;{ }^{a} 0.32_{-0.14}^{+0.10}$ & 0.00 \\
& $2_{2}^{+}$ & $<8^{a}$ & 0.96 \\
$2_{4 ; i}^{+}$ & $2_{1}^{+}$ & $0.019_{-0.019}^{+0.020}$ & 0.10 \\
$4_{3 ; i}^{+}$ & $2_{1}^{+}$ & $0.22_{-0.19}^{+0.09} ; 0.14(4)^{a}$ & 0.49 \\
& $2_{2}^{+}$ & $2.2_{-2.2}^{+1.4} ; 1.2(4)^{a}$ & 0.00 \\
& $2_{3 ; i}^{+}$ & $120_{-110}^{+50} ; 115(35)^{a}$ & 42.62 \\
& $4_{1}^{+}$ & $2.6_{-2.6}^{+1.6} ;{ }^{a} 1.8_{-1.5}^{+1.0}$ & 0.00 \\
\hline
\end{tabular}

${ }^{a}$ From Ref. 21]

normal states only, the "problematic" states $\left[0_{3}^{+}\left(n_{d}=2\right)\right.$ and $2_{5}^{+}\left(n_{d}=3\right)$ ] belong to class $\mathrm{B}$, and $0_{4}^{+}\left(n_{d}=3\right)$ belongs to class $\mathrm{C}$. The remaining "good" sphericalvibrator states $\left[0_{1}^{+}\left(n_{d}=0\right) ; 2_{1}^{+}\left(n_{d}=1\right) ; 4_{1}^{+}, 2_{2}^{+}\left(n_{d}=\right.\right.$ $2) ; 6_{1}^{+}, 4_{2}^{+}, 3_{1}^{+}\left(n_{d}=3\right)$ ] belong to class A. As mentioned, the spherical-vibrator interpretation is valid for most normal states in Fig. 1(a), but not all. We are thus confronted with a situation in which some states in the spectrum (assigned to class A) obey the predictions of U(5)DS, while other states (assigned to classes B and C) do not. These empirical findings signal the presence of a partial dynamical symmetry, U(5)-PDS.

The construction of Hamiltonians with U(5)-PDS follows the general algorithm 30, 40, by identifying operators which annihilate particular sets of $\mathrm{U}(5)$ basis states. In the present case, this leads to the following interaction:

$$
\hat{V}_{0}=r_{0} G_{0}^{\dagger} G_{0}+e_{0}\left(G_{0}^{\dagger} K_{0}+K_{0}^{\dagger} G_{0}\right),
$$

where $G_{0}^{\dagger}=\left[\left(d^{\dagger} d^{\dagger}\right)^{(2)} d^{\dagger}\right]^{(0)}, K_{0}^{\dagger}=s^{\dagger}\left(d^{\dagger} d^{\dagger}\right)^{(0)}$ and standard notation of angular momentum coupling is used. $\hat{V}_{0}$ of Eq. 6) is in normal-ordered form and satisfies

$$
\hat{V}_{0}\left|[N], n_{d}=\tau, \tau, n_{\Delta}=0, L\right\rangle=0,
$$

with $L=\tau, \tau+1, \ldots, 2 \tau-2,2 \tau$, for any choice of parameters $r_{0}$ and $e_{0}$. Equation (7) follows from the fact that the indicated states have $n_{d}=\tau$ and $n_{\Delta}=0$, hence do not contain a pair or a triplet of $d$-bosons coupled to $L=0$ and, as such, are annihilated by $K_{0}$ [4] and $G_{0}$ [4].

The states of Eq. (7), which include those of class A, form a subset of U(5) basis states, hence remain solvable eigenstates of the Hamiltonian

$$
\hat{H}_{\mathrm{PDS}}=\hat{H}_{\mathrm{DS}}+\hat{V}_{0}
$$

with good U(5) symmetry and energies given in Eq. (3) with $n_{d}=\tau$. It should be noted that while $\hat{H}_{\mathrm{DS}} \sqrt{2}$ is diagonal in the U(5)-DS chain (1), the $r_{0}$-term $\left(e_{0}\right.$-term) in $\hat{V}_{0}$ connects states with $\Delta n_{d}=0$ and $\Delta \tau=0, \pm 2, \pm 4, \pm 6$ $\left(\Delta n_{d}= \pm 1\right.$ and $\Delta \tau= \pm 1, \pm 3$ ). Accordingly, the remaining eigenstates of $\hat{H}_{\mathrm{PDS}}$ (8), in particular those of classes 
$\mathrm{B}$ and $\mathrm{C}$, are mixed with respect to $\mathrm{U}(5)$ and $\mathrm{SO}(5)$. The $\mathrm{U}(5)-\mathrm{DS}$ is therefore preserved in a subset of eigenstates, for any choice of parameters in $\hat{H}_{\mathrm{PDS}}$, but is broken in others. By definition, $\hat{H}_{\text {PDS }}$ exhibits U(5)-PDS. Cubic terms of the type present in $\hat{V}_{0}$, Eq. (6), were previously encountered in IBM studies of triaxiality [42, 43, signature splitting 39, 44, band anharmonicity 40, 45, and shape-coexistence [46, 47] in deformed nuclei. Such higher-order terms show up naturally in microscopicinspired IBM Hamiltonians derived by a mapping from self-consistent mean-field calculations [48, 49.

The effect of intruder levels can be studied in the framework of the interacting boson model with configuration mixing (IBM-CM) [50, 51]. The latter involves the space of normal states described by a system of $N$ bosons representing valence nucleon pairs, and the space of intruder states described by a system of $N+2$ bosons, accounting for particle-hole shell-model excitations. This procedure has been used extensively in describing coexistence phenomena in nuclei [52 55]. In the present study of ${ }^{110} \mathrm{Cd}$, the Hamiltonian in the normal sector is taken to be $\hat{H}_{\text {PDS }}$ of Eq. 8 , acting in a space of $N=7$ bosons. The Hamiltonian in the intruder sector is taken to be of $\mathrm{SO}(6)$-type [52,

$$
\hat{H}_{\text {intrud }}=\kappa \hat{Q} \cdot \hat{Q}+\Delta,
$$

acting in a space of $N=9$ bosons, with $\hat{Q}$ given in Eq. (4). A mixing term between the $[N]$ and $[N+2]$ boson spaces is defined as [52 55],

$$
\hat{V}_{\text {mix }}=\alpha\left[\left(s^{\dagger}\right)^{2}+\left(d^{\dagger} d^{\dagger}\right)^{(0)}\right]+\text { H.c. },
$$

where H.c. means Hermitian conjugate. The combined Hamiltonian for the two configurations has the form

$$
\hat{H}=\hat{H}_{\mathrm{PDS}}^{(N)}+\hat{H}_{\text {intrud }}^{(N+2)}+\hat{V}_{\text {mix }}^{(N, N+2)} .
$$

Here $\hat{\mathcal{O}}^{(N)}=\hat{P}_{N}^{\dagger} \hat{\mathcal{O}} \hat{P}_{N}$ and $\hat{\mathcal{O}}^{\left(N, N^{\prime}\right)}=\hat{P}_{N}^{\dagger} \hat{\mathcal{O}} \hat{P}_{N^{\prime}}$ for an operator $\hat{\mathcal{O}}$, with $\hat{P}_{N}$ a projection operator onto the $[N]$ boson space. In general, an eigenstate of $\hat{H}$,

$$
|\Psi\rangle=a\left|\Psi_{n}^{(N)}\right\rangle+b\left|\Psi_{i}^{(N+2)}\right\rangle,
$$

involves a mixture of normal $(n)$ and intruder $(i)$ components with $N$ and $N+2$ bosons, respectively. Similarly, the $E 2$ operator is defined as

$$
\hat{T}(E 2)=e_{B}^{(N)} \hat{Q}^{(N)}+e_{B}^{(N+2)} \hat{Q}^{(N+2)},
$$

with boson effective charges, $e_{B}^{(N)}$ and $e_{B}^{(N+2)}$.

The Hamiltonian of Eq. (11) has nine parameters. However, most of them only improve the fit to energies, but do not affect the structure of the states nor the calculated $E 2$ rates, which are the challenge in the $\mathrm{Cd}$ problem. The 6 parameters $\left(t_{i}, r_{0}, e_{0}\right)$ of $\hat{H}_{\mathrm{PDS}}$ (8) do not affect the $\mathrm{U}(5)$ purity of class-A states and, for small $\alpha$, the deviations from U(5)-DS in a few non-yrast states, is governed solely by the $r_{0}$ and $e_{0}$ terms. The comparison with the empirical data, discussed below, constitutes a stringent test for these PDS-based terms.

The spectrum and $B(E 2)$ values obtained with $\hat{H}$ 111 and $\hat{T}(E 2) \sqrt{13}$, are shown in Fig. 1(c) and Tables I-II. As seen, the IBM-PDS-CM calculation provides a good description of the empirical data in ${ }^{110} \mathrm{Cd}$. The normal states of class A retain good U(5) symmetry and quantum numbers, to a good approximation. Their $\left|\Psi_{n}^{(N)}\right\rangle$ part involves a single component with $n_{d}$ value as in Eq. (5a). The mixing with the intruder states is weak [small $b^{2}$ in Eq. [12)] and increases with $L$. Specifically, $b^{2}=0.9,2.2,3.6,5.9,4.6,6.1 \%$ for the $0_{1}^{+}, 2_{1}^{+}, 2_{2}^{+}, 4_{1}^{+}, 3_{1}^{+}, 4_{2}^{+}$states, respectively. The $6_{1}^{+}$ state experiences a somewhat larger mixing $\left(b^{2}=17.3 \%\right)$, consistent with its enhanced decay to the intruder $4_{3: i}^{+}$ state. The high degree of purity is reflected in the calculated $B(E 2)$ values for transitions between class A states which, as seen in Table I, are very similar to those of U(5)-DS. In contrast, the structure of the non-yrast states assigned originally to classes B and C, whose decay properties show marked deviations from the U(5)DS limit, changes dramatically. Specifically, the $0_{3}^{+}$and $0_{4}^{+}$states, which in the U(5)-DS classification are members of the two-phonon triplet and three-phonon quintuplet, interchange their character, and the $\mathrm{U}(5)$ decomposition of their $\left|\Psi_{n}^{(N)}\right\rangle$ parts peaks at $n_{d}=3$ and $n_{d}=2$, respectively. Similarly, the $2_{5}^{+}$and $2_{6}^{+}$states, which in the U(5)-DS classification are members of the three-phonon quintuplet and four-phonon octet, interchange their character, and the U(5) decomposition of their $\left|\Psi_{n}^{(N)}\right\rangle$ parts peaks at $n_{d}=4$ and $n_{d}=3$, respectively. The mixing with the intruder states is weak $\left(b^{2}=5.1 \%, 2.9 \%, 4.4 \%\right)$ for the $\left(0_{3}^{+}, 2_{5}^{+}, 2_{6}^{+}\right)$states, and somewhat larger $\left(b^{2}=18 \%\right)$ for the $0_{4}^{+}$state. The resulting calculated values: $B\left(E 2 ; 0_{3}^{+} \rightarrow 2_{1}^{+}\right)=0.25$, $B\left(E 2 ; 2_{5}^{+} \rightarrow 4_{1}^{+}\right)=0.19$ and $B\left(E 2 ; 2_{5}^{+} \rightarrow 2_{2}^{+}\right)=0.12$ W.u., are consistent with the measured upper limits: $7.9,5$ and $0.7_{-0.6}^{+0.5}$ W.u., respectively. The calculated decay $0_{4}^{+} \rightarrow 2_{2}^{+}$ is weaker than $0_{4}^{+} \rightarrow 2_{1}^{+}$, however, the observed dominant branching to the intruder $2_{3 ; i}^{+}$state is not reproduced. This may indicate a different structure for the $0_{4}^{+}$state (e.g., a $4 \mathrm{p}-6 \mathrm{~h}$ proton excitation as speculated in [21]). The dominant $E 2$ decays of the $2_{6}^{+}$state (not shown in Fig. 1) are predicted to be $B\left(E 2 ; 2_{6}^{+} \rightarrow 0_{4}^{+}\right)=24.28$, $B\left(E 2 ; 2_{6}^{+} \rightarrow 4_{1}^{+}\right)=15.73, B\left(E 2 ; 2_{6}^{+} \rightarrow 2_{2}^{+}\right)=9.27$ and $B\left(E 2 ; 2_{6}^{+} \rightarrow 4_{3 ; i}^{+}\right)=3.56$ W.u.

A few monopole transition rates are experimentally known in ${ }^{110} \mathrm{Cd}$ [56], expressed in terms of the quantity $\rho(E 0)=\frac{\langle f|\hat{T}(E 0)| i\rangle}{e R^{2}}$, with $R=1.2 A^{1 / 3} \mathrm{fm}$. The corresponding $E 0$ operator in the IBM-CM, can be transcribed [57] in the form $\hat{T}(E 0)=\left(e_{n} N^{\prime}+e_{p} Z\right) \eta\left(\hat{n}_{d}^{(N)}+\right.$ $\left.\hat{n}_{d}^{(N+2)}\right)$, where $N^{\prime}(Z)$ are neutron (proton) numbers and $e_{p}=2 e_{n}=e$. The measured [56] and calculated (in curly brackets) strengths are given by $\rho^{2}\left(E 0 ; 0_{2 ; i}^{+} \rightarrow\right.$ $\left.0_{1}^{+}\right) \cdot 10^{3}<31\{0.75\}, \rho^{2}\left(E 0 ; 2_{3 ; i}^{+} \rightarrow 2_{1}^{+}\right) \cdot 10^{3}=9(8)\{10\}$, $\rho^{2}\left(E 0 ; 4_{3 ; i}^{+} \rightarrow 4_{1}^{+}\right) \cdot 10^{3}=106_{-91}^{+98}\{36\}$. The calculated 
strengths, obtained with $\eta=0.063$, reproduce the measured values, within the quoted error bars. The calculation predicts $\rho^{2}(E 0) \cdot 10^{3} \sim 10$ for $0_{4}^{+} \rightarrow 0_{2 ; i}^{+}$and $2_{4 ; i}^{+} \rightarrow 2_{2}^{+}$ $E 0$ transitions, which have not been measured so far.

In summary, we have addressed a key question concerning the phonon structure of states in $\mathrm{Cd}$ isotopes. Our results suggest that the vibrational interpretation of ${ }^{110} \mathrm{Cd}$ can be salvaged by introducing a boson Hamiltonian that mixes particular phonon states while keeping the mixing with coexisting intruder levels weak. The proposed scheme relies on a partial dynamical U(5) symmetry, in which most low-lying normal states in ${ }^{110} \mathrm{Cd}$ maintain their spherical-vibrational character and only a few specific non-yrast states exhibit a departure from $\mathrm{U}(5)$, in line with the empirical data. Work currently in progress appears to indicate that the same PDS-based approach can be implemented also in other neutron-rich $\mathrm{Cd}$ isotopes, at least as an appropriate starting point for further refinements.

This work is supported in part (A.L. and N.G.) by the Israel Science Foundation (Grant 586/16) and (J.E.G.R.) by the Spanish Ministerio de Economía y Competitividad and the European regional development fund (FEDER) under Projects No. FIS2014-53448-C2-2-P and by Consejería de Economía, Innovación, Ciencia y Empleo de la Junta de Andalucía (Spain) under Groups FQM-370.
[1] A. Bohr, Mat. Fys. Medd. Dan. Vid. Selsk. 26 no 14, (1952).

[2] A. Bohr and B. R. Mottelson, Mat. Fys. Medd. Dan. Vid. Selsk. 27 no 16, (1953).

[3] A. Bohr and B. R. Mottelson, Nuclear Structure. II Nuclear Deformations (Benjamin, New York, 1975).

[4] F. Iachello and A. Arima, The Interacting Boson Model (Cambridge University Press, Cambridge, 1987).

[5] D. Bonatsos, Interacting Boson Models of Nuclear Structure, (Clarendon, Oxford, 1988).

[6] R. F. Casten, Nuclear Structure from a Simple Perspective (Oxford University Press, Oxford, 2000).

[7] K. Heyde, Basic Ideas and Concepts in Nuclear Physics (Institute of Physics, Bristol, 2004).

[8] A. Aprahamian, D. S. Brenner, R. F. Casten, R. L. Gill and A. Piotrowski, Phys. Rev. Lett. 59, 535 (1987).

[9] M. Délèze, S. Drissi, J. Jolie, J. Kern and J. P. Vorlet, Nucl. Phys. A 554, 1 (1993).

[10] G. Gneuss and W. Greiner, Nucl. Phys. A 171, 449 (1971).

[11] K. Heyde and J. L. Wood, Rev. Mod. Phys. 83, 1467 (2011).

[12] H. W. Fielding et al., Nucl. Phys. A 281, 389 (1977).

[13] J. Kumpulainen et al., Phys. Rev. C 45, 640 (1992).

[14] K. Heyde, P. Van Isacker, M. Waroquier, G. Wenes and M. Sambataro, Phys. Rev. C 25, 3160 (1982).

[15] M. Délèze et al., Nucl. Phys. A 551, 269 (1993).

[16] P. E. Garrett et al., Phys. Rev. C 75, 054310 (2007).

[17] R. F. Casten, J. Jolie, H. G. Börner, D. S. Brenner, N. V. Zamfir, W.-T. Chou and A. Aprahamian, Phys. Lett. B 297, 19 (1992).

[18] M. Kadi, N. Warr, P. E. Garrett, J. Jolie and S. W. Yates, Phys. Rev. C 68, 031306(R) (2003).

[19] P. E. Garrett, K. L. Green and J. L. Wood, Phys. Rev. C 78, 044307 (2008).

[20] P. E. Garrett and J. L. Wood, J. Phys. G 37, 064028 (2010); 069701 (2010).

[21] P. E. Garrett et al., Phys. Rev. C 86, 044304 (2012).

[22] J. C. Batchelder et al., Phys. Rev. C 86, 064311 (2012).

[23] J.C. Batchelder et al., Phys. Rev. C 89, 054321 (2014).

[24] E. A. Coello Pérez and T. Papenbrock, Phys. Rev. C 92, 064309 (2015).

[25] P. E. Garrett, J. Phys. G 43, 084002 (2016).

[26] F. M. Prados-Estévez et al., Phys. Rev. C 95, 034328 (2017).
[27] K. Nomura and J. Jolie, Phys. Rev. C 98, 024303 (2018).

[28] A. Leviatan, Prog. Part. Nucl. Phys. 66, 93 (2011).

[29] F. Iachello, Lie algebras and applications (Springer, Berlin Heidelberg, 2006).

[30] Y. Alhassid and A. Leviatan, J. Phys. A 25, L1265 (1992).

[31] A. Leviatan, Phys. Rev. Lett. 77, 818 (1996).

[32] A. Leviatan, A. Novoselsky and I. Talmi, Phys. Lett. B 172, 144 (1986).

[33] P. Van Isacker, Phys. Rev. Lett. 83, 4269 (1999).

[34] A. Leviatan and P. Van Isacker, Phys. Rev. Lett. 89, 222501 (2002).

[35] A. Arima and F. Iachello, Ann. Phys. (N.Y.) 99 (1976) 253.

[36] G. Gürdal and F.G. Kondev, Nucl. Dat. Sheets 113, 1315 (2012).

[37] A. Leviatan and I. Sinai, Phys. Rev. C 60, 061301(R) (1999).

[38] R. F. Casten, R. B. Cakirli, K. Blaum and A. Couture Phys. Rev. Lett. 113, 112501 (2014).

[39] A. Leviatan, J.E. García-Ramos and P. Van Isacker, Phys. Rev. C 87, 021302(R) (2013).

[40] J.E. García-Ramos, A. Leviatan and P. Van Isacker, Phys. Rev. Lett. 102, 112502 (2009).

[41] I. Talmi, in Symmetries in Nuclear Structure, (A. Vitturi and R. F. Casten Eds.), World Scientific p. 10 (2004).

[42] K. Heyde, P. Van Isacker, M. Waroquier and J. Moreau, Phys. Rev. C 29, 1420 (1984).

[43] N. V. Zamfir and R. F. Casten, Phys. Lett. B 260, 265 (1991).

[44] D. Bonatsos, Phys. Lett. B 200, 1 (1988).

[45] J. E. García-Ramos, J. M. Arias and P. Van Isacker, Phys. Rev. C 62, 064309 (2000).

[46] A. Leviatan and D. Shapira, Phys. Rev. C 93, 051302(R) (2016).

[47] A. Leviatan and N. Gavrielov, Phys. Scr. 92, 114005 (2017).

[48] K. Nomura, N. Shimizu, D. Vretenar, T. Nikšić and T. Otsuka, Phys. Rev. Lett. 108, 132501 (2012).

[49] K. Nomura, R. Rodríguez-Guzmán and L. M. Robledo, Phys. Rev. C 87, 064313 (2013).

[50] P. D. Duval and B. R. Barrett, Phys. Lett. B 100, 223 (1981); Nucl. Phys. A 376, 213 (1982).

[51] M. Sambataro and G. Molnar, Nucl. Phys. A 376, 201 (1982). 
[52] K. Heyde, J. Jolie, H. Lehmann, C. De Coster and J. L. Wood, Nucl. Phys. A 586, 1 (1995).

[53] R. Fossion, K. Heyde, G. Thiamova and P. Van Isacker, Phys. Rev. C 67, 024306 (2003).

[54] J. E. García-Ramos, V. Hellemans and K. Heyde, Phys. Rev. C 84, 014331 (2011).
[55] J.E. García-Ramos and K. Heyde, Phys. Rev. C 89 , 014306 (2014); Phys. Rev. C 92, 034309 (2015).

[56] B. Jigmeddorj et al., Eur. Phys. J. A 52, 36 (2016).

[57] S. Zerguine, P. Van Isacker and A. Bouldjedri Phys. Rev. C 85, 034331 (2012). 